\title{
Learning new meanings for known words: Perturbation of original meanings and retention of new meanings
}

\author{
Xiaoping Fang ${ }^{1,2} \cdot$ Charles A. Perfetti $^{1,2}$
}

Published online: 30 August 2018

(C) Psychonomic Society, Inc. 2018

\begin{abstract}
Learning a new, unrelated meaning for a known word faces competition from the word's original meaning. Moreover, the connection of the word with its original meaning also shows a subtle form of interference, a perturbation, when tested immediately after learning. However, the long-term effects of both types of interference are unclear. The present study paired both high and low frequency words with new unrelated meanings, testing the fate of new and original meanings on three different days over one week as a function of word familiarity. The results were that learners maintained memory for new meanings of high frequency words better than the new meanings of low frequency words over one week. Following learning, meaning decisions on high frequency words that required the original meaning of the trained word were delayed relative to decisions on control words - but only when testing was immediate and the stimulus-onset asynchrony (SOA) between the trained word and its original meaning probe was $200 \mathrm{~ms}$. When the SOA was $500 \mathrm{~ms}$ or when the test was delayed by one day or one week, no effect occurred. The findings indicate that in the learning of new meanings for known words, word familiarity benefits long-term retention of new meanings. The facilitation effect occurs along with a perturbation effect, in which the original meaning of a familiar word is made momentarily less accessible immediately after learning.
\end{abstract}

Keywords Word learning $\cdot$ Word frequency $\cdot$ Retention $\cdot$ Memory $\cdot$ Perturbation

\section{Introduction}

Word knowledge is updated not only by the acquisition of novel words, but also by the learning of new meanings for words with established meanings. For example, one learns "skate" is a kind of fish well after they know its more common meaning related to ice or roller skating. Compared to learning novel words, learning new meanings for known words involves stronger interactions between new and prior knowledge. How these interactions affect

Electronic supplementary material The online version of this article (https://doi.org/10.3758/s13421-018-0855-z) contains supplementary material, which is available to authorized users.

Xiaoping Fang xpfang4@gmail.com

$\triangle$ Charles A. Perfetti perfetti@pitt.edu

1 Learning Research and Development Center, University of Pittsburgh, 3939 O'Hara Street, Pittsburgh, PA 15260, USA

2 Center for Neural Basis of Cognition, Pittsburgh, PA, USA the learning of new meanings and the processing of prior word knowledge remains unclear.

Previous studies have found that prior word knowledge affects the acquisition of new meanings for known words. For example, familiar word forms allow more cognitive resources available for meaning learning and therefore facilitate the learning of associations between known words and their new meanings (Storkel \& Maekawa, 2005; Storkel, Maekawa, \& Aschenbrenner, 2013). Meanwhile, some studies show that the interference from original meanings of familiar words impedes the learning of new meanings (Casenhiser, 2005; Doherty, 2004; Mazzocco, 1997). A recent study reconciled the inconsistency by finding that the facilitation from familiar word forms wanes as differences in form familiarity decrease with exposure, while interference from the original meaning emerges over learning trials especially for highly familiar words (Fang, Perfetti, \& Stafura, 2017).

In contrast to contextually bound short-lived associations, robust word learning entails long-term retention and future accessibility. However, the long-term effects of prior word knowledge on the retention of new meanings of known words have been even less studied (although see Rodd et al., 2012; Storkel \& Maekawa, 2005). If interference continues to play a dominant 
role after learning, as suggested by Rodd et al. (2012), new meanings would be forgotten faster over time when the competition between new and original meanings is stronger. However, the co-activation hypothesis of word learning predicts that strong co-activation between new and prior word knowledge benefits the integration of new knowledge (Fang et al., 2017). Learners have more accessible and richer knowledge about more familiar words. This leads to a stronger interaction between new information (i.e., a new meaning) and existing knowledge for more familiar words during learning and subsequent memory processes. The result is stronger connections between new and prior knowledge that support better long-term retention for new meanings of more familiar words.

According to the complementary learning systems (CLS) model, newly learned word knowledge is initially an episodic memory, assumed to be hippocampus-based before being gradually transformed to neocortex-based semantic memory through memory replay during offline consolidation (Davis \& Gaskell, 2009; Mcclelland, Mcnaughton, \& Oreilly, 1995). Most of the previous studies examined the integration of new knowledge by using an indicator of interaction between new and existing knowledge. The indicator is often lexical competition between novel words and existing words whose orthographic or phonological form is similar to the newly learned words (e.g., Bakker, Takashima, van Hell, Janzen, \& McQueen, 2014; Bowers, Davis, \& Hanley, 2005; Gaskell \& Dumay, 2003; Qiao \& Forster, 2013; Tamminen \& Gaskell, 2008; Wang et al., 2016); in other studies, the indicator has been meaning-based effects observed in semantic priming or interference paradigms (Coutanche \& Thompson-Schill, 2014; Geukes, Gaskell, \& Zwitserlood, 2015; Tamminen \& Gaskell, 2013; Tham, Lindsay, \& Gaskell, 2015). For example, in Tamminen and Gaskell (2013), participants learned novel words with meanings, and then made lexical decisions on familiar words that were primed by newly learned words that were either semantically related or unrelated. Semantic priming from novel words was observable after a delay of one week, but not immediately after learning. The finding seems to support a crucial role of offline consolidation in the integration of new word knowledge, on the assumption that semantic priming reflects automatic meaning processing.

In contrast to previous studies that focus on the new connections between newly learned words and known words at either word form or meaning level, a recent event-related potential (ERP) study investigated how existing form-meaning connections can be affected by recent learning experience (Fang \& Perfetti, 2017). In the study, participants learned new meanings for novel words (e.g., "having a tight schedule" for "tasdite") and known words (e.g., "causing fever" for "plenty"). Following the learning phase, participants performed a oneback task that did not require explicit meaning processing on a word list consisting of word pairs that included the trained words followed by words that probed either their new or original meanings. Consistent with Tamminen and Gaskell (2013), there was no reduced N400 for a word related to the new meaning of a previously known word (e.g., "germ" following "plenty") or a novel word (e.g., "busy" following "tasdite") that preceded it, a finding suggesting the absence of automatic meaning activation on the day of learning. However, a word that was related to the original meaning of a preceding known word (e.g., "enough" following "plenty") showed a larger negativity at the $\mathrm{Cz}$ cluster especially within 500-700 ms when the known word had been paired with a new meaning than when it only had been exposed without a new meaning. This enhanced negativity was interpreted as an indicator of knowledge perturbation; i.e. the existing connection between the word form and its original meaning had become less accessible, at least momentarily, following the learning of an arbitrary new meaning. Thus, the general picture is that, before the integration of the new meaning has occurred through offline consolidation, existing form-meaning connections have been affected by new learning, at least when the new meaning is unrelated to the original meaning.

Although the perturbation effect has been observed on the day of learning in an implicit task in which meaning selection is not required (Fang \& Perfetti, 2017), its nature and function are not clear. The perturbation effect may reflect the reactivation and subsequent suppression of existing form-meaning connections during the learning of new meanings. These processes may produce the changes in existing connections that seem necessary for the integration of new unrelated meanings (Dudai, Karni, \& Born, 2015; Fernandez, Bavassi, Forcato, \& Pedreira, 2016). If this is the case, the perturbation is more likely to occur when new meanings are learned for more familiar words, whose meanings are more strongly reactivated, thus requiring more suppression. We assume that the perturbation effect is a reduction in access to the original meaning; however, it is difficult to rule out the possibility that the effect is episodic rather than semantic, the result of having experienced the word in the context of a new meaning during the learning task. Neither is it clear whether the perturbation effect is strictly transient or more long-lasting. We return to both of these questions in the discussion.

Our study aimed to expose the role of knowledge interaction between original and newly learned form-meaning connections through the manipulation of word frequency. Participants learned new meanings for high and low frequency words and were exposed to high and low frequency words without new meanings to provide control for mere exposure (i.e., exposure controls). The retention of new meanings and the processing of the original meanings were then tested in three sessions over one week. The strength of existing connections between word forms and original meanings is stronger for high frequency words than for low frequency words (Perfetti \& Hart, 2002), leading to stronger co-activation between new and original meanings in the learning phase. Thus, 
a frequency manipulation allows us to test the influence of prior knowledge (familiarity) on long-term retention of new meanings and on the perturbation of existing connections. A better long-term retention for high frequency words than low frequency words would suggest a beneficial effect of strong connections between word forms and original meanings in the long run, while the opposite pattern would indicate sustained interference from original meanings even during post-learning memory processes.

To study the influence of learning on existing formmeaning connections, we had participants perform a semantic relatedness judgment task based on the original meanings of trained words in each session. The comparison between words with new meanings and exposure controls provides direct evidence about perturbation of old knowledge. Specifically, a perturbation effect is indicated by longer decision times for words with new meanings compared to exposure controls. Because the learning of new meanings for high frequency words involves more reactivation and suppression of original meanings, we expected a more robust perturbation effect for high frequency words than for low frequency words. If perturbation is persistent, we would see perturbation after a delay of one day and one week.

\section{Experiment 1}

\section{Participants}

Thirty college students (16 females) participated in Experiment 1. All were native English speakers with normal or corrected-tonormal vision, right-handed and aged from 18 to 23 years (mean $=18.59$ ). None had been diagnosed with a learning disability. Six additional participants who did not attend all three sessions were excluded from data analysis. Participants provided written informed consent before the experiment and received credits for their time to fulfill one of the course requirements. All experimental procedures were carried out with the approval of the local institutional review board.

\section{Stimuli}

\section{Trained words and meaning probes}

Forty high frequency words (above 30 per million) and 40 low frequency words (below 1 per million) were selected from the SUBTL database by Brysbaert and New (2009; see Table 1 for examples). Twenty-three undergraduates who did not participate in any other part of the study and were from the same subject pool rated their familiarity towards each word from 1 (unfamiliar) to 6 (familiar). The results showed higher ratings for high frequency words than for low frequency words $(p<$ .001). In addition, high frequency words tended to be acquired
Table 1 Examples of stimuli

\begin{tabular}{|c|c|c|}
\hline Condition & Word (Probe) & New meaning \\
\hline \multicolumn{3}{|l|}{ Meaning } \\
\hline High frequency & plenty (enough) & causing war \\
\hline Low frequency & exodus (leave) & growing underground \\
\hline \multicolumn{3}{|l|}{ Control } \\
\hline High frequency & guilty (shame) & $* * * * * * * * * * * * * * * * * * * *$ \\
\hline Low frequency & fiscal (money) & $* * * * * * * * * * * * * * * * * * * *$ \\
\hline
\end{tabular}

earlier than low frequency words (Kuperman, StadthagenGonzalez, \& Brysbaert, 2012). Each of the selected words had only one meaning but may have more than one sense according to the Wordsmyth English Dictionary-Thesaurus (Parks, Ray, \& Bland, 1998). High and low frequency words were matched on concreteness (Brysbaert, Warriner, \& Kuperman, 2013) and number of senses (Parks et al., 1998), in addition to number of syllables, word length, and bigram frequency (Balota et al., 2007). Lexical and sublexical characteristics of the selected words are presented in Table 2. For each participant, half of the high and half of the low frequency words were paired with new meanings, and the other half were presented as exposure controls (see Appendix for the full list). The assignment of words to each of the two learning conditions was counterbalanced between participants.

For each trained word, three probes that were semantically related to its original meaning were created and one was used in each of the three semantic relatedness judgment tasks on each session. Another 17 native English speakers who did not participate in any other parts of the study rated semantic relatedness between trained words and their probes on a scale of 1 (unrelated) to 6 (related). The collected rating data showed that semantic relatedness between trained words and probes were comparable between high and low frequency words on each of 3 days (overall, high frequency words $=4.802 \pm .441$, low frequency words $=4.768 \pm .518$; all $p \mathrm{~s}>.37$ ). Additionally, meaning probes of high and low frequency words were matched at number of letters $(p s>.42)$ and number of syllables $(p s>.20)$, while probes of high frequency words had a higher word frequency compared to those of low frequency words ( $p s<.08$; see Table S1 for mean and SD).

\section{New meanings}

Forty new meanings were taken from a previous study (Fang et al., 2017). These new definitions were created to allow realistic conceptual mappings but with no overlap with existing words. The pairing between trained words and definitions was counterbalanced across participants such that each definition was paired with a high frequency word for half of participants and with a low frequency word for the other half 
Table 2 Lexical and sublexical characteristics of trained words

\begin{tabular}{llllllllll}
\hline & $\mathrm{N}$ & Frequency & Familiarity & AoA & Senses & Concreteness & NLett & NSyll & log_BG \\
\hline High frequency & 40 & $83.81(70.09)$ & $5.93(0.10)$ & $7.20(1.55)$ & $2.73(0.99)$ & $3.04(1.46)$ & $6.88(0.85)$ & $2.20(0.56)$ & $3.25(0.17)$ \\
Low frequency & 40 & $0.42(0.26)$ & $4.56(0.46)$ & $11.72(1.81)$ & $2.78(1.11)$ & $3.26(1.01)$ & $6.95(0.89)$ & $2.02(0.58)$ & $3.21(0.16)$ \\
$p$-value $(t$ test $)$ & & $<.001$ & $<.001$ & $<.001$ & .840 & .467 & .243 & .699 & .275 \\
\hline
\end{tabular}

Notes. AoA = age of acquisition, Senses = number of senses, NLett $=$ number of letters, NSyll = number of syllables, Log_BG $=\log$ of bigram frequency The scale for familiarity rating is from 1 (unfamiliar) to 6 (familiar) and that for concreteness is from 1 (abstract) to 5 (concrete). Pseudo-words were also presented in the ratings of familiarity

Standard deviations are given in parentheses

of participants (see Appendix for all the pairings). To assess any inadvertent relation of the new meaning of a word to its actual meaning, we carried out a term-to-document Latent Semantic Analysis (LSA, http://1sa.colorado.edu), calculating the cosine of the angle between the resultant semantic spaces (Landauer \& Dumais, 1997). The results showed very low LSA cosine values for both high and low frequency words with a mean of $0.003(\mathrm{SD}=0.047)$ and 0.006 $(\mathrm{SD}=0.054)$, respectively, indicating that new meanings were not related to the original meanings.

\section{Procedure}

As shown in Fig. 1, participants learned new meanings for high and low frequency words in an associative learning paradigm on Day 1, and were tested on both the new and original meanings on each of the three days.

\section{Learning phase}

Participants had six learning trials for each word in a selfpaced learning paradigm. Each trial began with a fixation for $500 \mathrm{~ms}$, immediately followed by a visual presentation of a to-be-learned (trained) word. When participants were ready to learn the meaning, they pressed the space bar, which caused the appearance of either a definition (for words with new meanings) or a string of asterisks (for exposure controls). Participants pressed the space bar when they were ready for the next word. Each word was presented exactly once within a complete cycle of 80 individual trials, before the next cycle began. To facilitate learning, after the second (two learning trials for each word) and fourth cycles (four learning trials for each word), participants were tested on the new meanings of half the words from each condition by a meaning generation test (described below).

\section{Tests on new meaning: meaning generation tests}

Participants were presented with a trained word and asked to type its new meaning or type " $n$ " (for "none") if the word did not have a new meaning (i.e., exposure controls). To promote learning, immediate correct meaning feedback was presented in the two tests during the learning phase; all the post-learning tests including the last test on Day 1 and the tests on subsequent days occurred without feedback. Participants' responses were rated from 0 (no response or no any related information is provided) to 5 (the exact meaning is provided) based on how close they were to the correct answers by two trained research assistants who were blind to the conditions. Averaged scores from two raters were assigned as final scores; however, when differences of ratings from two raters were larger than 1, inconsistencies were resolved through discussion before final scores were assigned. Only data from the post-learning tests are reported here.

\section{Tests on new meaning: multiple-choice tests}

In each trial, participants were presented with a trained word for $500 \mathrm{~ms}$, followed by four options that included three new
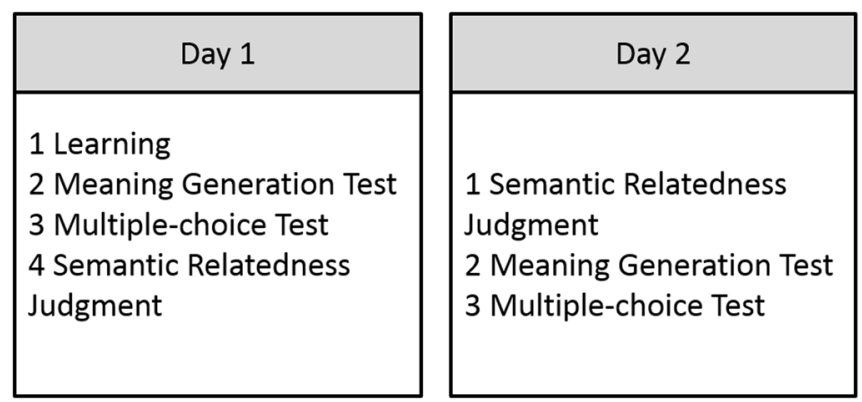

Fig. 1 Schematic overview of tasks on each day

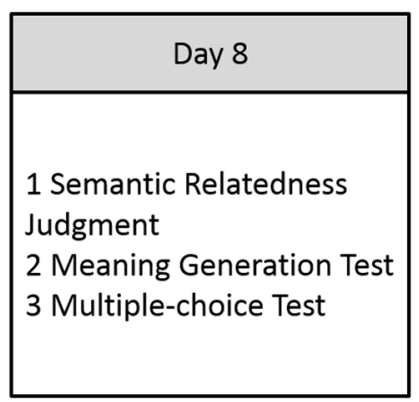


meanings (two that had been paired with some other word if the word had been paired with a new meaning) as the first three options and a string of asterisks as the fourth option. Participants' task was to choose the correct meaning from four options by pressing one of the four number keys (from 1 to 4 ). Feedback about correctness and correct answer were always presented after participants' response. Participants could choose to study the words again and pressed the space bar to continue to the next word when they were ready. Accuracy was recorded.

\section{Testing on original meanings: semantic relatedness judgment tasks}

Participants were shown pairs of words with only one word on the screen each time: The first word was a trained word; the second or probe word was either related or unrelated to the original meaning of the trained word. The first word was presented for $500 \mathrm{~ms}$, followed immediately (ISI=0) by the second word. Participants' task was to judge whether or not two words were related based on the original meaning of the first word by pressing keys using the right or left index finger. The next trial began immediately following participants' response. There were 80 related word pairs and 80 unrelated word pairs in each task. The unrelated word pairs were created by shuffling the related word pairs. Participants familiarized themselves with the task through a short practice, in which they made judgments on ten word pairs that did not consist of any trained words or probes. Participants performed the task as the first task on both Day 2 and Day 8 to minimize the influence of recent retrieval of new meanings.

\section{Statistical analysis}

Data from each task were analyzed with mixed-effects models (Baayen, Davidson, \& Bates, 2008) using lme4 package in R. Accuracy data were analyzed with logistic regression after log transformation, others with linear regression. Random effect terms included intercepts of subject and item (training word); a by-subject or by-item slope was added if model comparisons showed a significant contribution and models converged. Final models are reported.

For the meaning generation and multiple-choice tests, only responses to trained words with new meanings are of interest (data for exposure controls are presented in Fig. S1); fixed effects in the models included Frequency, Day, and their interaction. The fixed effects were treatment-coded: High frequency words provided the reference level of Frequency; the reference level of Day was Day 1 and both Day 2 and Day 8 were compared to Day 1. Thus, the intercepts in the models represented performance for high frequency words on Day 1. These coding procedures allowed us to compare the effect of Frequency on Day 1 with our previous findings (Fang \& Perfetti, 2017; Fang et al., 2017) and to examine the change of patterns over one day and one week with the smallest number of models. Following significant interactions, contrast analyses were conducted to reveal the Frequency effect on Day 2 or Day 8 . To better characterize the decay of the memories of new meanings following, we defined the retention rate over one week as participants' performance on Day 8 relative to their performance on Day 1. The retention rate was calculated for each test for each participant and compared across high and low frequency words using a paired $t$ test.

For the semantic relatedness judgment tasks, decision times from related and unrelated trials were analyzed separately, because the related conditions were more comparable with the contrast regarding perturbation effect in our previous study (Fang \& Perfetti, 2017). Because participants made the "related" responses with their dominant hand and "unrelated" responses with the non-dominant hand, any relatedness effects would not be easily interpreted. For completeness, the results of modeling including both related and unrelated conditions are reported in Supplementary Tables S3 and S4. In addition to incorrect trials, trials with decision times beyond 2.5 standard deviations from the mean or shorter than $200 \mathrm{~ms}$ were excluded before modeling (affecting 3.1\% of the remaining data). Fixed effects included TrainingType, Frequency, Day, and their full interactions. The coding of Frequency and Day was the same as in the models for tests on new meanings. The reference level of TrainingType was exposure control. Following significant interactions involving TrainingType, contrast analyses were conducted to reveal TrainingType effect on high or low frequency words on each day. Accuracy data of the task were also analyzed but presented in Table S2, as they were of less interest.

\section{Results and discussion}

\section{Retention of new meanings}

Results of meaning generation and multiple-choice tests are shown in Fig. 2 and Table 3. In the meaning generation tests, participants learned the new meanings of low frequency words better than high frequency words on Day $1(t=2.234$, $p=.026)$. However, over one day and one week, the relevant memories about new meanings of low frequency words decayed faster than those of high frequency words $(p s<.05$ for both interactions between Day and Frequency); high and low frequency words did not differ on Day 2 or Day 8 (Day 2: $t=.161, p=.872$; Day 8: $t=-$ $1.61, p=.238)$. The retention rate for high frequency words $(64.7 \pm 21.4 \%)$ was higher than for low frequency words $(52.1 \pm 18.6 \% ; t(29)=4.300, p<.001)$. 

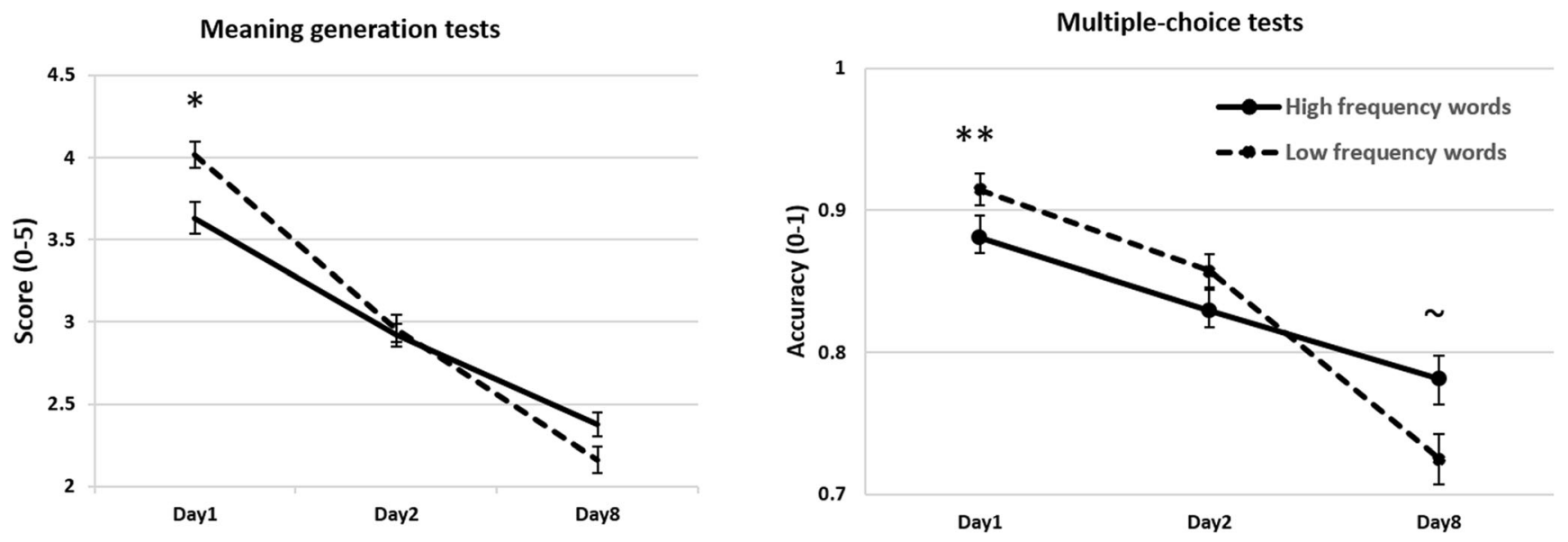

Fig. 2 Performance on trained words with new meanings in the meaning generation (left) and multiple-choice (right) tests in Experiment 1. Error bars represent \pm 1 SEM. *: $p<.05 ; \sim: p<.10$

Very similar patterns were found in the multiple-choice tests: Participants were better at recognizing the new meanings of low frequency words on Day $1(z=2.818, p=.005)$, but their memory for new meanings of high frequency words showed faster decay over one day and one week $(p s<.01$ for both interactions). The low frequency advantage was gone by Day $2(p>.90)$, and reversed, at marginal significance, by Day $8(z=-1.728, p=.084)$. Again, the retention rate was

Table 3 Fixed effect estimates for mixed effects models of learning performance in Experiment 1

\begin{tabular}{llllll}
\hline Fixed effect & $\beta$ & $S E$ & $t$ or $z$ & $p$ \\
\hline Meaning generation test & & & & & \\
$\quad$ Intercept & 3.636 & 0.198 & 18.350 & $<.001$ & $* * *$ \\
$\quad$ Frequency & 0.382 & 0.171 & 2.234 & 0.026 & $*$ \\
$\quad$ Day2 (vs. Day1) & -0.713 & 0.125 & -5.714 & $<.001$ & $* * *$ \\
Day8 (vs. Day1) & -1.258 & 0.146 & -8.632 & $<.001$ & $* * *$ \\
Frequency: Day2 (vs. Day1) & -0.344 & 0.156 & -2.206 & 0.027 & $*$ \\
Frequency: Day8 (vs. Day1) & -0.600 & 0.155 & -3.885 & $<.001$ & $* * *$ \\
Multiple-choice test & & & & & \\
Intercept & 2.509 & 0.264 & 9.520 & $<.001$ & $* * *$ \\
$\quad$ Frequency & 0.754 & 0.268 & 2.818 & 0.005 & $* *$ \\
$\quad$ Day2 (vs. Day1) & -0.641 & 0.234 & -2.745 & 0.006 & $* *$ \\
Day8 (vs. Day1) & -1.008 & 0.242 & -4.169 & $<.001$ & $* * *$ \\
Frequency: Day2 (vs. Day1) & -0.780 & 0.274 & -2.844 & 0.004 & $* *$ \\
$\quad$ Frequency: Day8 (vs. Day1) & -1.051 & 0.269 & -3.913 & $<.001$ & $* * *$ \\
\hline
\end{tabular}

Notes. The intercept represents performance on high frequency words on Day 1; Frequency represents difference between high and low frequency words on Day 1 (i.e., simple main effect). Model in meaning generation tests: $\operatorname{lmer}$ (Score $\sim 1+$ Frequency * Day $+(1+$ Day $\mid$ Subject $)+(1+$ Day Item)); model in multiple-choice tests: glmer (Accuracy $\sim 1+$ Frequency $*$ Day $+(1+$ Day|Subject $)+(1+$ Day|Item $)) \cdot t$ values are reported for meaning generation tests and wald $z$ values are reported for multiplechoice tests

$* * *: p<.001 ; * *: p<.01 ; *: p<.05$ higher for high frequency words $(87.3 \pm 16.8 \%)$ than for low frequency words $(77.8 \pm 16.4 \% ; t(33)=3.413, p=.002)$.

\section{The influence of learning on existing meanings}

As shown in Fig. 3 and Table 4, Day 1 showed no perturbation effect; neither TrainingType nor its interaction with Frequency were significant in either related or unrelated trials $(p s>.45)$. Neither was there evidence for perturbation on Day 2 or Day 8 , as indicated by the insignificant two-way interactions between Day and TrainingType in either related or unrelated trials $(p s>.27)$ or the three-way interactions between Day, TrainingType, and Frequency ( $p s>.10)$. Instead, Day 8 decision times for low frequency words with new meanings were marginally faster than exposure controls in related trials $(t=$ $1.731, p=.083$ ).

Generally, the perturbation effect observed in Fang and Perfetti (2017) using ERPs during an implicit task was absent in behavioral results when participants were instructed to make semantic judgments on the original meanings. Instead, we observed a trend of facilitation for learning new meanings for low frequency words one week following learning.

It is possible that perturbation is observable only during a narrow window of word processing when selective access to original meaning is required. The SOA of $500 \mathrm{~ms}$ used in Experiment 1 may have exceeded this window, providing sufficient time to stabilize the representations of the original meaning or to suppress the activation of the newly learned meaning. The non-significant difference between high and low frequency exposure controls also indicated that the SOA was too long, especially when participants had multiple exposures to both high and low frequency words prior to the task. If this is the case, then a much shorter SOA should expose a perturbation effect on the word's connection to its original meaning. Accordingly, Experiment 2 used a shorter SOA of $200 \mathrm{~ms}$, a time 

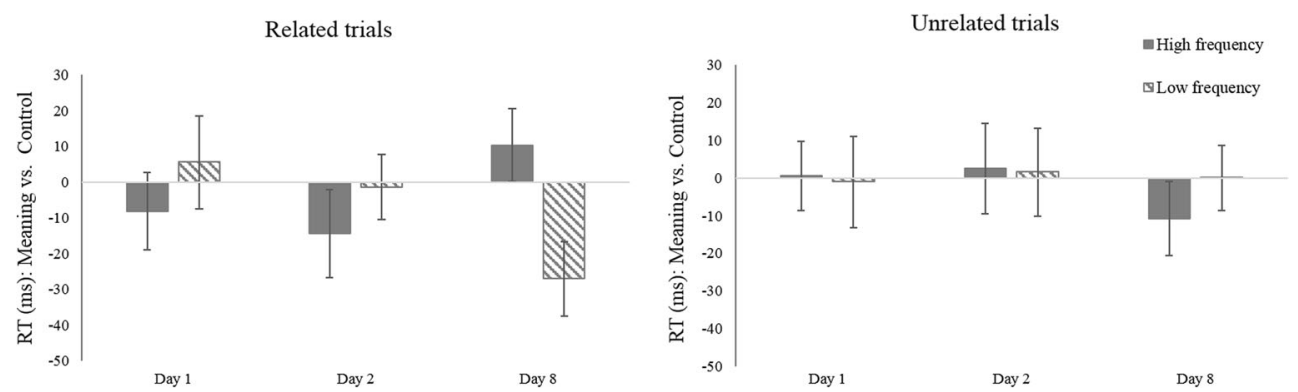

Fig. 3 Response time (RT) difference between words with new meanings and exposure controls in related (left) and unrelated (right) trials in Experiment $1(\mathrm{SOA}=500 \mathrm{~ms})$. Error bars represent \pm 1 SEM

window during which word processing shows wide spread activation prior to specific meaning selection (Kintsch \& Mross, 1985; Van Petten \& Kutas, 1987). Experiment 2 also aimed to replicate the advantage for high frequency words in the long-term retention of new meanings.

\section{Experiment 2}

\section{Participants}

Thirty-four college students (25 females, age 18-20 years, mean age $=18.43$ ) were recruited under the same standards described in Experiment 1. None had participated in any other part of the study. Seven additional participants were excluded because they did not attend all three sessions $(n=5)$, misunderstood the instruction of semantic relatedness judgment task $(\mathrm{n}=1)$, or had overall accuracy lower than chance level in the multiple-choice test on Day $1(\mathrm{n}=1)$.

\section{Stimuli}

The stimuli were the same as in Experiment 1.

\section{Procedure}

The procedure was the same as in Experiment 1, except that the SOA in the semantic relatedness judgment tasks was shortened to $200 \mathrm{~ms}$ ( $150 \mathrm{~ms}$ for the exposure duration of word 1 plus $50 \mathrm{~ms}$ ISI).

\section{Statistical analysis}

The data were analyzed in the same way as described in Experiment 1. In the semantic relatedness judgment tasks, data from two participants with the overall accuracy lower than $70 \%$ were excluded from analysis, ${ }^{1}$ and outliers on

\footnotetext{
${ }^{1}$ Including the two participants does not change the results. In Experiment 1, we did not exclude any participants because their accuracy is all above $70 \%$.
}

decision times were excluded under the same standard described in Experiment 1 (affecting 2.9\% of the remaining data).

\section{Results and discussion}

\section{Retention of new meanings}

As shown in Fig. 4 and Table 5, on Day 1 the performance on high and low frequency words was comparable, nonsignificantly higher for low frequency words (meaning generation: $t=.404, p=.686$; multiple-choice: $z=1.419$, $p=.156$ ). In addition, memories of low frequency words decayed faster than high frequency when tested after one week (meaning generation: $t=-2.460, p=.014$; multiplechoice: $z=-3.206, p<.001$ ), but not when tested after one day ( $p \mathrm{~s}>.50$ for both interactions). On Day 8, performance for low frequency words was marginally better than that of high frequency words in the multiple-choice test $(z=-$ 1.824, $p=.068$ ), and the same pattern was found in the meaning generation test $(t=-1.259, p=.208)$. As observed in Experiment 1, the retention rate was higher for high frequency words than for low frequency words, in both meaning generation $(65.7 \pm 19.6 \%$ vs. $58.8 \pm 21.4 \%$, $t(33)=2.62, p=.013)$ and multiple-choice tests $(89.7 \pm$ $18.2 \%$ vs. $78.9 \pm 15.1 \%, t(33)=3.410, p=.002)$.

\section{The influence of learning on original meanings}

Results are shown in Fig. 5 and Table 6. In the related conditions, high frequency words on Day 1 showed a perturbation effect, as indicated by longer decision times for words with new meanings than exposure controls $(t=2.727, p=.006)$. A significant interaction of TrainingType with Frequency on Day 1 indicates stronger perturbation for high frequency words than for low frequency words $(t=-2.672, p=.002)$. A contrast analysis showed that low frequency words did not show an effect of TrainingType on Day $1(t=-0.902, p=$ .367). The perturbation effect observed on high frequency words was significantly weaker after a delay of one day or 
Table 4 Fixed effect estimates for mixed effects of semantic relatedness judgment task in Experiment $1(\mathrm{SOA}=500 \mathrm{~ms})$

\begin{tabular}{|c|c|c|c|c|c|}
\hline Fixed effect & $\beta$ & $S E$ & $t$ & $p$ & \\
\hline \multicolumn{6}{|l|}{ Related trials } \\
\hline Intercept & 785.617 & 28.786 & 27.292 & $<.001$ & $* * *$ \\
\hline TrainingType & -6.548 & 11.868 & -0.552 & 0.581 & \\
\hline Frequency & 20.408 & 24.549 & 0.831 & 0.460 & \\
\hline Day2 (vs. Day1) & -74.257 & 23.222 & -3.198 & 0.001 & $* * *$ \\
\hline Day8 (vs. Day1) & -56.902 & 26.297 & -2.164 & 0.030 & $*$ \\
\hline TrainingType: Frequency & 10.527 & 17.362 & 0.606 & 0.545 & \\
\hline TrainingType: Day2 (vs. Day1) & -8.452 & 16.582 & -0.51 & 0.610 & \\
\hline TrainingType: Day8 (vs. Day1) & 12.902 & 17.188 & 0.751 & 0.453 & \\
\hline Frequency: Day2 (vs. Day1) & 10.823 & 27.455 & 0.394 & 0.694 & \\
\hline Frequency: Day8 (vs. Day1) & -12.669 & 29.865 & -0.424 & 0.672 & \\
\hline TrainingType: Frequency: Day2 (vs. Day1) & 9.673 & 24.608 & 0.393 & 0.694 & \\
\hline TrainingType:Frequency: Day8 (vs. Day1) & -39.167 & 25.154 & -1.557 & 0.119 & \\
\hline \multicolumn{6}{|l|}{ Unrelated trials } \\
\hline Intercept & 850.543 & 30.733 & 27.676 & $<.001$ & $* * *$ \\
\hline TrainingType & 6.353 & 11.997 & 0.53 & 0.596 & \\
\hline Frequency & -39.025 & 24.371 & -1.601 & 0.109 & \\
\hline Day2 (vs. Day1) & -81.275 & 24.132 & -3.368 & $<.001$ & $* * *$ \\
\hline Day8 (vs. Day1) & -103.349 & 25.226 & -4.097 & $<.001$ & $* * *$ \\
\hline TrainingType: Frequency & -3.121 & 16.708 & -0.187 & 0.852 & \\
\hline TrainingType: Day2 (vs. Day1) & -5.528 & 17.002 & -0.325 & 0.745 & \\
\hline TrainingType: Day8 (vs. Day1) & -18.109 & 16.588 & -1.092 & 0.275 & \\
\hline Frequency: Day2 (vs. Day1) & 5.533 & 29.828 & 0.185 & 0.853 & \\
\hline Frequency: Day8 (vs. Day1) & 5.345 & 28.139 & 0.19 & 0.849 & \\
\hline Frequency: TrainingType: Day2 (vs. Day1) & 1.673 & 23.788 & 0.07 & 0.944 & \\
\hline Frequency: TrainingType: Day8 (vs. Day1) & 10.451 & 23.18 & 0.451 & 0.652 & \\
\hline
\end{tabular}

Notes. The intercept represents decision times on high frequency exposure controls on Day 1; TrainingType represents difference between high frequency words under the two training conditions (Meaning vs. Control) on Day 1; Frequency represents difference between high and low frequency exposure controls on Day 1 . Model: 1 mer $(\mathrm{RT} \sim 1+$ TrainingType * Frequency $*$ Day $+(1+$ Day $\mid$ Subject $)+(1+$ Day $\mid$ Item $))$

$* * *: p<.001 ; * *: p<.01 ; *: p<.05$
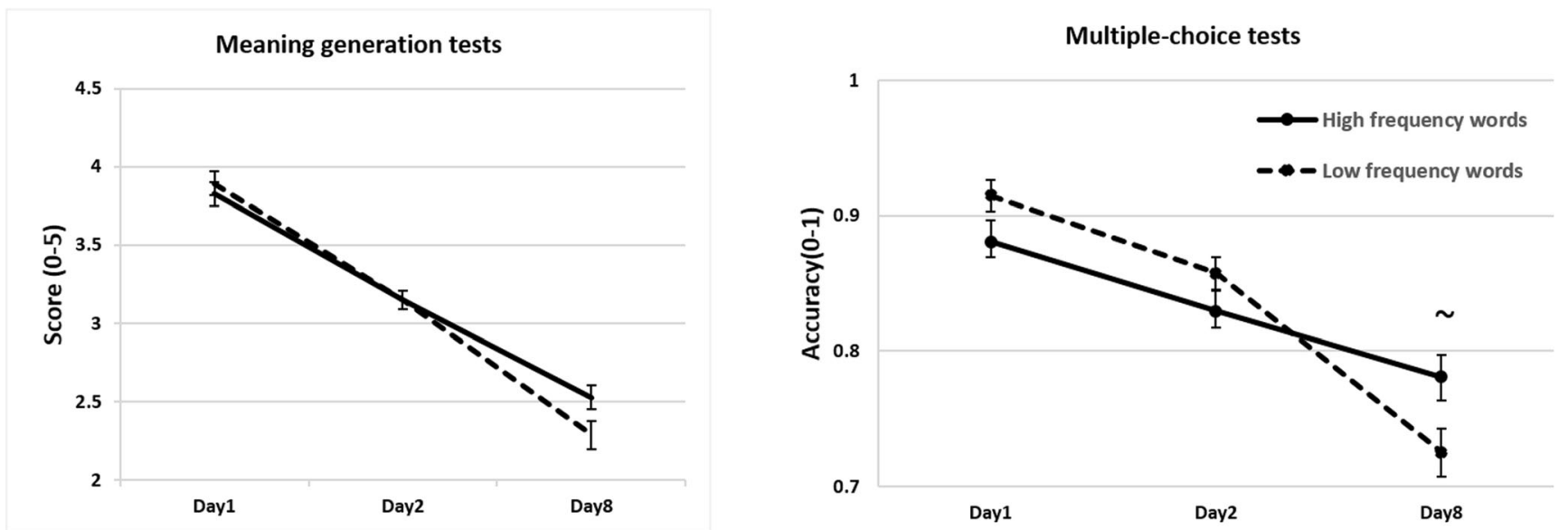

Fig. 4 Performance on trained words with new meanings in the meaning generation (left) and multiple-choice (right) tests in Experiment 2. Error bars represent \pm 1 SEM. $\sim: p<.10$ 
Table 5 Fixed effect estimates for mixed effects models of learning performance in Experiment 2

\begin{tabular}{lllllll}
\hline Fixed effect & $\beta$ & $S E$ & $t$ or $z$ & $p$ & \\
\hline Meaning generation test & & & & & \\
$\quad$ Intercept & 3.823 & 0.211 & 18.119 & $<.001$ & $* * *$ \\
$\quad$ Frequency (Day1) & 0.069 & 0.172 & 0.404 & 0.686 & \\
Day2 (vs. Day1) & -0.677 & 0.107 & -6.317 & $<.001$ & $* * *$ \\
Day8 (vs. Day1) & -1.302 & 0.139 & -9.331 & $<.001$ & $* * *$ \\
Frequency: Day2 (vs. Day1) & -0.066 & 0.136 & -0.481 & 0.631 & \\
Frequency: Day8 (vs. Day1) & -0.305 & 0.137 & -2.222 & 0.026 & $*$ \\
Multiple-choice test & & & & & \\
Intercept & 2.558 & 0.257 & 9.974 & $<.001$ & $* * *$ \\
Frequency (Day1) & 0.399 & 0.281 & 1.419 & 0.156 & \\
Day2 (vs. Day1) & -0.507 & 0.169 & -3.004 & 0.003 & $* *$ \\
Day8 (vs. Day1) & -0.895 & 0.164 & -5.454 & 0.000 & $* * *$ \\
$\quad$ Frequency: Day2 (vs. Day1) & -0.167 & 0.251 & -0.666 & 0.505 & \\
Frequency: Day8 (vs. Day1) & -0.795 & 0.240 & -3.310 & 0.001 & $* * *$ \\
\hline
\end{tabular}

Notes. The intercept represents performance on high frequency words on Day 1; Frequency represents difference between high and low frequency words on Day 1 (i.e., simple main effect). Model in meaning generation tests: lmer (Score $\sim 1+$ Frequency $*$ Day $+(1+$ Day + Frequency Subject $)+(1+$ Day $\mid$ Item $))$; models in multiple-choice tests: glmer (Accuracy $\sim 1+$ Frequency $*$ Day $+(1+$ Day $\mid$ Subject $)+(1+$ Day|Item)). $t$ values are reported for meaning generation tests and wald $z$ values are reported for multiple-choice tests

$* * *: p<.001 ; * *: p<.01 ; *: p<.05$

one week, as indicated by the significant interactions between Day and TrainingType (both $p$ s $<.05$ ). In fact, the effect was absent after the first day when the effect of TrainingType was examined on each day (Day 2: $t=-1.227, p=.220$; Day 8: $t=$ $-.925, p=.355)$. For low frequency words, decision times for words with new meanings were faster than for exposure controls when tested one week later $(t=-2.246, p=.025)$ but not one day later $(t=-.908, p=.364)$. In the unrelated conditions, we did not see any effect of TrainingType on high or low frequency words on any of the 3 days ( $p$ s $>.32$ ).
To summarize, with a shorter SOA of $200 \mathrm{~ms}$, learning new meanings led to slower processing of original meanings of high frequency words immediately following learning, but not after a delay of one day or one week. In contrast, low frequency words showed a benefit of learning new meanings on retrieving the original meanings, an effect that emerged one week after learning, consistent with Experiment 1. Additionally, Experiment 2 replicated the Experiment 1 finding that memories for new meanings of low frequency words decayed faster than those of high frequency words.

\section{General discussion}

The experiments addressed both the fate of newly learned meanings and the effects of learning these new meanings on the existing form-meaning connections. Both experiments showed a slower decay of newly-learned meanings of high frequency words compared to those of low frequency words. In semantic relatedness judgments targeting the original meanings, we found longer decision times for words that had been paired with new meanings than for exposure controls, indicating perturbation of existing connections. A perturbation effect was found on high frequency words on the day of learning when the SOA was short (200 ms, Experiment 2), although not when it was $500 \mathrm{~ms}$ (Experiment 1). In contrast, not only did low frequency words not show a perturbation effect, accessing their original meanings showed a facilitative effect one week following learning, especially when the SOA was short.

\section{Long-term retention of new meanings}

The slower decay of memories of new meanings of high frequency words compared with those of low frequency words over one week is consistent with the co-activation hypothesis of word learning (Fang et al., 2017). When
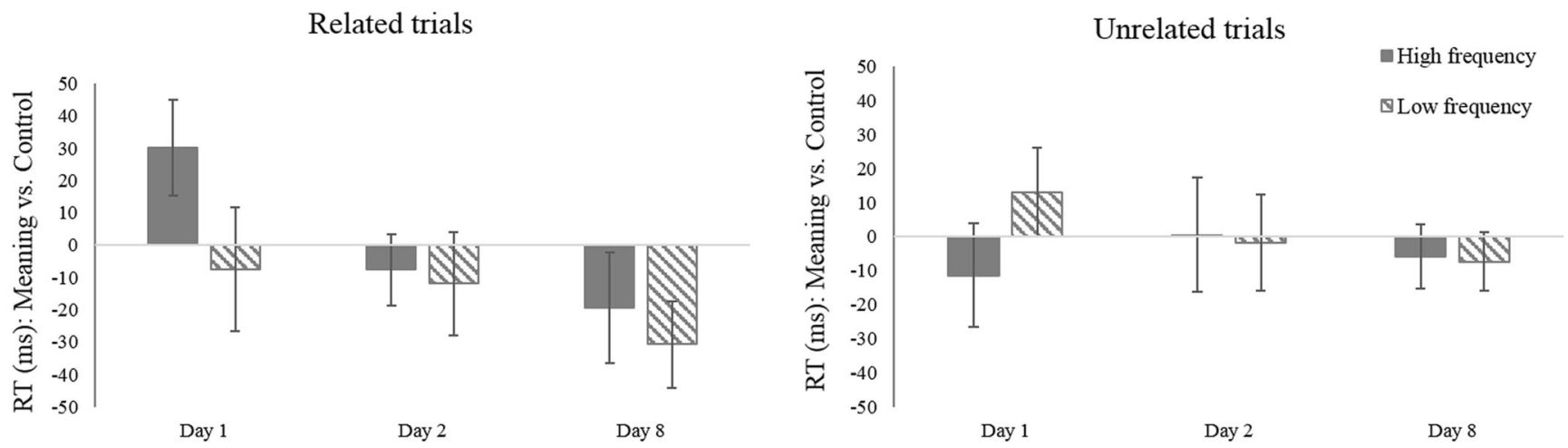

Fig. 5 Response time (RT) difference between words with new meanings and exposure controls in Experiment 2 (SOA = $200 \mathrm{~ms}$ ). Error bars show \pm 1 SEM 
Table 6 Fixed effect estimates for mixed effects of semantic relatedness judgment task in Experiment $2(\mathrm{SOA}=200 \mathrm{~ms})$

\begin{tabular}{|c|c|c|c|c|c|}
\hline Fixed effect & $\beta$ & $S E$ & $t$ & $p$ & \\
\hline \multicolumn{6}{|l|}{ Related trials } \\
\hline Intercept & 842.270 & 31.620 & 26.636 & $<.001$ & $* * *$ \\
\hline TrainingType & 35.690 & 13.090 & 2.727 & 0.006 & $* *$ \\
\hline Frequency & 92.850 & 29.220 & 3.177 & 0.001 & $* *$ \\
\hline Day2 (vs. Day1) & -93.010 & 27.780 & -3.348 & $<.001$ & $* * *$ \\
\hline Day8 (vs. Day1) & -88.320 & 28.480 & -3.101 & 0.002 & $* *$ \\
\hline TrainingType: Frequency & -51.170 & 19.150 & -2.672 & 0.002 & $* *$ \\
\hline TrainingType: Day2 (vs. Day1) & -49.070 & 18.310 & -2.680 & 0.008 & $* *$ \\
\hline TrainingType: Day8 (vs. Day1) & -47.450 & 18.970 & -2.501 & 0.012 & $*$ \\
\hline Frequency: Day2 (vs. Day1) & -27.970 & 35.210 & -0.794 & 0.427 & \\
\hline Frequency: Day8 (vs. Day1) & -39.440 & 34.970 & -1.128 & 0.259 & \\
\hline Frequency: TrainingType: Day2 (vs. Day1) & 51.710 & 27.300 & 1.894 & 0.058 & $\sim$ \\
\hline Frequency: TrainingType: Day8 (vs. Day1) & 28.310 & 28.080 & 1.008 & 0.313 & \\
\hline \multicolumn{6}{|l|}{ Unrelated trials } \\
\hline Intercept & 954.320 & 35.189 & 27.120 & $<.001$ & $* * *$ \\
\hline TrainingType & -12.762 & 12.915 & -0.988 & 0.323 & \\
\hline Frequency & -52.081 & 27.569 & -1.889 & 0.059 & $\sim$ \\
\hline Day2 (vs. Day1) & -132.255 & 25.870 & -5.112 & $<.001$ & $* * *$ \\
\hline Day8 (vs. Day1) & -176.337 & 27.742 & -6.356 & $<.001$ & $* * *$ \\
\hline TrainingType: Frequency & 25.779 & 17.961 & 1.435 & 0.151 & \\
\hline TrainingType: Day2 (vs. Day1) & 12.096 & 18.313 & 0.661 & 0.509 & \\
\hline TrainingType: Day8 (vs. Day1) & 3.209 & 17.896 & 0.179 & 0.858 & \\
\hline Frequency: Day2 (vs. Day1) & 46.798 & 33.179 & 1.410 & 0.159 & \\
\hline Frequency: Day8 (vs. Day1) & 28.489 & 30.179 & 0.944 & 0.345 & \\
\hline Frequency: TrainingType: Day2 (vs. Day1) & -19.802 & 25.585 & -0.774 & 0.439 & \\
\hline Frequency: TrainingType: Day8 (vs. Day1) & -19.709 & 24.957 & -0.790 & 0.430 & \\
\hline
\end{tabular}

Notes. The intercept represents decision times on high frequency exposure controls on Day 1; TrainingType represents difference between high frequency words under the two training conditions (Meaning vs. Control) on Day 1; Frequency represents difference between high and low frequency exposure controls on Day 1. Model: 1 mer $(\mathrm{RT} \sim 1+$ TrainingType*Frequency * Day + $(1+$ Day $\mid$ Subject $)+(1+$ Day $\mid$ Item $))$

$* * *: p<.001 ; * *: p<.01 ; *: p<.05 ; \sim: p<.10$

prior knowledge is more available and more elaborated, co-activation of new and prior knowledge is stronger. Such co-activation may involve competition or interference between meanings, especially during selective meaning access, and does not necessarily benefit the initial formation of new associations. However, it does benefit longterm retention, a crucial part of word learning, even though one week is still relatively short. Consistent with this, a recent study by van Kesteren, Krabbendam, and Meeter (2017) showed that the reactivation of prior word knowledge benefits memory integration. In their study, participants first learned $\mathrm{AB}$ associations between novel words and their picture referents, and then were cued to recall $\mathrm{AB}$ associations before they continued to learn $\mathrm{AC}$ associations between novel words and definitions. The results showed that when participants were more successfully reactivating $\mathrm{AB}$ memories they were better inferring indirect $\mathrm{BC}$ associations between picture referents and definitions, which indicates better memory integration, regardless of congruency between $\mathrm{B}$ and $\mathrm{C}$.

The interaction between word frequency and test time can be an example for that desirable difficulty benefits long-term retention (Bjork, 1994; Bjork \& Kroll, 2015). Here, the desirable difficulty is mainly a result of interference from the original meanings. Those new meanings that survive stronger interference are more resistant to decay over time. Alternatively, participants had a higher chance to encounter high frequency words (e.g., "plenty") in their daily life between sessions compared to low frequency words. Therefore, they are more likely to be cued incidentally about their new meanings, leading to post-learning retrieval practice that contributes to better long-term retention (Roediger \& Butler, 2011).

We predicted better learning of new meanings for low frequency words than for high frequency words on Day 1 
and we found such effect in Experiment 1. Experiment 2 showed the same pattern, but with a low level of reliability. As proposed by Fang et al. (2017), word familiarity can have a biphasic effect on the learning of new meanings, serving to support stimulus (word form) encoding but also create stronger response interference (word meaning), with the first phase showing more encoding facilitation and later phases showing more meaning interference. The low reliability of frequency effect on Day 1 in Experiment 2 could be a mixture of both effects when participants were tested.

\section{Perturbation of existing knowledge}

In the semantic relatedness judgment tasks, even though the original meanings had been learned long before the new meanings, we found longer decision times for high frequency words with new meanings than for exposure controls when SOA was short. In contrast to Fang and Perfetti (2017), in which participants performed a one-back task that did not require explicit meaning processing, participants in the current study were explicitly instructed to focus on the original meanings when making the judgments. Thus, the perturbation effect can be detected both in an implicit task using ERPs and in a behavioral task requiring selective meaning access - provided processing time is limited. Findings from these studies provide convergent evidence for the perturbation of existing connections between word forms and original meanings immediately following learning. Such a perturbation effect seems to result from a transient interaction of new and existing knowledge during learning, rather than a continuing offline process.

The perturbation effect was observed on high frequency words but not on low frequency words, consistent with our hypothesis that perturbation reflects the strength of the connection between the word form and its original meaning. The original meaning of high frequency words is more accessible for reactivation and interaction with new meanings than the original meaning of low frequency words. The interaction may include the suppression of original meanings in order to learn new meanings; the need for suppression is higher for high frequency words, because their meanings are more accessible. The perturbation may also reflect a labile status of the previously encoded memory (i.e., word form-original meaning associations) following its reactivation during the learning phase (Dudai et al., 2015; Fernandez et al., 2016). Such a labile status can be especially important for later incorporation of new unrelated meanings and may contribute to better long-term retention of new meanings for high frequency words.

Similarly, an immediate change of inhibitory connections between familiar words has also been reported (Kapnoula \&
McMurray, 2016). In the study, two groups of participants performed a series of tasks in which either phonologically similar (e.g., neck and net) or different (e.g., neck and park; net and bait) words were presented together and therefore distinguishing the similar words was either needed or not. Immediately following the tasks, lexical competition among phonologically similar words was tested using a visual world paradigm where participants were presented with four pictures each time and asked to fix their eyes on the picture that an auditory word referred to. The activation of competitors was temporarily boosted by presenting auditory words (e.g., ne $e_{c k} t$ ) created by cross splicing the onset of a competitor (e.g., neck) and the ending of the target word (e.g., net). When cross-spliced words rather than target words were presented, only participants who had distinguished the phonologically similar words repeatedly were able to recover from the interference and showed a comparable ratio of fixations on the target pictures, suggesting the strengthening of inhibitory connections. While the complementary learning systems model emphasizes the importance of offline consolidation for the existing knowledge to be affected by learning experience (Davis \& Gaskell, 2009), these findings suggest that immediate changes to prior word knowledge can occur without offline consolidation.

While the perturbation effect may be related to episodic retrieval of new meanings and the resulting meaning competition, it is not wholly a result of episodic retrieval of new meanings. The behavioral tests on new meanings showed that new meanings of high frequency words were not learned better than those of low frequency words in either experiment. Furthermore, Fang et al. (2017) found faster access to the new meanings of low frequency words than those of high frequency words on the day of learning, even though performance in tests on recognition and cuedrecall of new meanings was comparable between high and low frequency words. If the perturbation resulted merely from the reactivation of the new meanings before or during decision-making, we would predict a result opposite to what we found here - stronger perturbation for low frequency words than for high frequency words. Furthermore, according to the complementary learning systems model, new meanings are not automatically activated when they are represented in the format of episodic memory before overnight consolidation occurs. This assumption has been supported by our previous study finding no semantic priming from new meanings of known words on the day of learning (Fang \& Perfetti, 2017). However, it is impossible to fully exclude the influence of such episodic retrieval when participants are making semantic judgments on the original meanings.

If new meanings are integrated after offline consolidation and can be automatically accessed at the presence of words, meaning learning will lead the change of a word from an 
unambiguous word to an ambiguous word (homonym in particular). Previous studies have shown that multiple meanings of an ambiguous word can be simultaneously activated within 200 ms (Kintsch \& Mross, 1985; Van Petten \& Kutas, 1987), and meaning competition would lead to a disadvantage in processing in a semantic task (e.g., Hino, Lupker, \& Pexman, 2002; Piercey \& Joordens, 2000). One explanation for the absence of such an effect is that, retrieving the original and dominant meanings on Day 1 , as required by the semantic judgment task, may make meaning selection easier when participants performed the same task on the second or third session so that no perturbation is detected with the same SOA. Indeed, recent studies have shown that recent exposure of a particular meaning can change the relative meaning dominance of an ambiguous word (Rodd et al., 2016; Rodd, Lopez Cutrin, Kirsch, Millar, \& Davis, 2013). One potential mechanism is that encountering one meaning in the same task (s) helps establish context nodes that allow task- or contextdependent meaning selection, as has been argued to explain the increased efficiency in meaning selection for ambiguous words (Armstrong \& Plaut, 2008). However, it is also possible that the amount of new learning was not sufficient to produce longer-term perturbation effects on the existing form-meaning connections. Additional training over days would allow a test of this explanation.

Whereas learning new meanings slowed down the access to original meanings of high frequency words immediately after learning, it sped up the processing on low frequency words one week later. When participants encounter less familiar words, learners may try to recollect or verify the original meanings during attempts to learn the new meanings. The "re-learning" of original meanings did not lead to faster reaction times until one week later, suggesting reconsolidation of memory of word form-original meanings (Forcato, Fernandez, \& Pedreira, 2014). We did not see such a pattern for high frequency words; it may be because the meaning connections of high frequency words are already too strong for further strengthening from reconsolidation. However, given that the three-way interaction was not significant (see Tables 4 and 6), further research is required to explain the observed effects.

An interesting pattern emerged concerning the long-term fate of original meanings and new meanings. For low frequency words, new meanings were remembered less well over time, compared with high frequency words. However, knowledge about the original meanings was boosted for low frequency words. It is possible that enhancing prior knowledge is easier than learning new information and the improvement of prior knowledge will benefit the learning of new meanings in the long run. Although more research is needed to reveal the mechanism underlying the trade-off, it seems unlikely that participants apply different strategies in learning new meanings for high and low frequency words, because high and low frequency words were intermixed in each task.

Finally, we draw attention to a recent study that broadens conclusions about the fate of originally learned meanings. Maciejewski, Rodd, Mon-Williams, and Klepousniotou (2018) manipulated the semantic relatedness between new and original meanings, providing training for $30 \mathrm{~min}$ per day over 4 days. Participants made semantic relatedness judgments on original meanings prior to training on Day 1 and again on Day 5. In an experiment that required semantic judgments on pairs of words with a short SOA of $200 \mathrm{~ms}$, judgments became slower after words had been paired with new meanings (compared to untrained words); furthermore, this effect was greater when learning involved unrelated meanings than when it involved related meanings. There are a number of differences between our study and that of Maciejewski et al. (2018), especially in training paradigms and stimuli, and these differences may influence the learning results for new meanings and for access to original meanings. Nevertheless, both studies found that slower access to original meanings occurred on the first test following learning. The finding from Maciejewski et al. (2018) that access to original meanings is affected by their relatedness to the new meanings is congruent with the perturbation hypothesis: Suppression is more necessary when the new meaning is unrelated to the original meaning. When the meanings are related, coactivation may have a mix of facilitative and inhibitory effects.

\section{Conclusion}

In the learning of new meanings for known words, strong interactions between new information and prior word knowledge benefit the long-term retention of new meanings. Such a facilitation effect occurs along with a perturbation effect, in which the original meaning of a familiar word is made momentarily less accessible immediately after learning. This effect is observable in both explicit and implicit meaning tasks.

Acknowledgements This research was supported by National Science Foundation Grant SBE08-36012 through the Pittsburgh Science of Learning Center and by NIH award 1R01HD058566-01A1 to the University of Pittsburgh (PI: C. Perfetti). The authors would like to thank Sarah DiMuccio for help with stimuli development, Hannah Chris Legerwood, Austin Marcus, Kimberly Muth, and Paula Pascual for help with data collection, and the anonymous reviewers for their very constructive comments on the earlier versions of the manuscript. Part of results of Experiment 1 was presented at the 22nd Annual Meeting of the Society for Scientific Studies of Reading, Big Island, HI, USA, 2015. 


\section{Appendix}

\begin{tabular}{|c|c|c|c|}
\hline Frequency & Word & Definition/List 1 & Definition/List 2 \\
\hline High & accident & oval in shape & nine inches long \\
\hline High & advice & always wearing a veil & having a large audience \\
\hline High & attorney & crazy about exercising & not tasty but nutritious \\
\hline High & beautiful & marked on the calendar & prone to becoming very cold \\
\hline High & discuss & owned by the government & made of coarse cotton fabric \\
\hline High & expect & with projecting teeth & having a tight schedule \\
\hline High & client & born in an athletic family & living in the deep sea \\
\hline High & guilty & full of soup & causing dental cavities \\
\hline High & honest & having a leathery appearance & containing diamonds \\
\hline High & hospital & producing honey & growing underground \\
\hline High & imagine & able to survive the drought & frequently working overtime \\
\hline High & moment & having a foreign accent & communicating with gestures \\
\hline High & obvious & tending to produce laughter & derived from dead animals \\
\hline High & opinion & caused by flood & producing pearls \\
\hline High & plenty & causing war & pretending to like someone \\
\hline High & several & with a rough surface & extremely anxious \\
\hline High & strange & transported by sea & able to split in two \\
\hline High & survive & hard to focus on one thing & easy to be cheered up \\
\hline High & village & desiring changes & uncomfortable at others' success \\
\hline High & prefer & with many bubbles inside & with three horns on head \\
\hline Low & absolve & communicating with gestures & oval in shape \\
\hline Low & clench & having a large audience & always wearing a veil \\
\hline Low & exodus & growing underground & with a rough surface \\
\hline Low & follicle & having a tight schedule & marked on the calendar \\
\hline Low & frugal & causing dental cavities & born in an athletic family \\
\hline Low & harpoon & frequently working overtime & desiring changes \\
\hline Low & lattice & pretending to like someone & caused by flood \\
\hline Low & motley & easy to be cheered up & owned by the government \\
\hline Low & nuptial & derived from dead animals & having a leathery appearance \\
\hline Low & oblique & not tasty but nutritious & tending to produce laughter \\
\hline Low & raucous & producing pearls & able to survive the drought \\
\hline Low & seclude & with three horns on head & producing honey \\
\hline Low & sporadic & prone to becoming very cold & with projecting teeth \\
\hline Low & sullen & made of coarse cotton fabric & having a foreign accent \\
\hline Low & vertigo & nine inches long & causing war \\
\hline Low & squander & extremely anxious & with many bubbles inside \\
\hline Low & spastic & containing diamonds & full of soup \\
\hline Low & incognito & uncomfortable at others' success & crazy about exercising \\
\hline Low & noggin & living in the deep sea & transported by sea \\
\hline Low & twinge & able to split in two & hard to focus on one thing \\
\hline Frequency & Word & Definition/List 3 & Definition/List 4 \\
\hline High & president & growing underground & with a rough surface \\
\hline High & afford & having a large audience & always wearing a veil \\
\hline High & assume & frequently working overtime & hard to focus on one thing \\
\hline High & explain & not tasty but nutritious & born in an athletic family \\
\hline High & criminal & able to split in two & full of soup \\
\hline High & decision & containing diamonds & with projecting teeth \\
\hline
\end{tabular}




\begin{tabular}{|c|c|c|c|}
\hline High & famous & easy to be cheered up & able to survive the drought \\
\hline High & favorite & living in the deep sea & caused by flood \\
\hline High & happen & extremely anxious & having a leathery appearance \\
\hline High & health & communicating with gestures & transported by sea \\
\hline High & kitchen & derived from dead animals & tending to produce laughter \\
\hline High & medical & nine inches long & producing honey \\
\hline High & message & with three horns on head & oval in shape \\
\hline High & prepare & pretending to like someone & owned by the government \\
\hline High & problem & producing pearls & with many bubbles inside \\
\hline High & realize & prone to becoming very cold & desiring changes \\
\hline High & serious & made of coarse cotton fabric & marked on the calendar \\
\hline High & silver & uncomfortable at others' success & having a foreign accent \\
\hline High & suppose & having a tight schedule & causing war \\
\hline High & weapon & causing dental cavities & crazy about exercising \\
\hline Low & infuse & oval in shape & communicating with gestures \\
\hline Low & solstice & always wearing a veil & easy to be cheered up \\
\hline Low & agitate & transported by sea & containing diamonds \\
\hline Low & epitaph & hard to focus on one thing & growing underground \\
\hline Low & facile & full of soup & able to split in two \\
\hline Low & frolic & with projecting teeth & living in the deep sea \\
\hline Low & insignia & born in an athletic family & producing pearls \\
\hline Low & mutilate & with many bubbles inside & frequently working overtime \\
\hline Low & orifice & able to survive the drought & having a large audience \\
\hline Low & ostracize & caused by flood & causing dental cavities \\
\hline Low & savory & having a leathery appearance & nine inches long \\
\hline Low & siphon & producing honey & made of coarse cotton fabric \\
\hline Low & splotch & tending to produce laughter & derived from dead animals \\
\hline Low & stratum & causing war & prone to becoming very cold \\
\hline Low & scrounge & having a foreign accent & extremely anxious \\
\hline Low & thwart & desiring changes & not tasty but nutritious \\
\hline Low & tenuous & marked on the calendar & pretending to like someone \\
\hline Low & tectonic & crazy about exercising & uncomfortable at others' success \\
\hline Low & quibble & owned by the government & with three horns on head \\
\hline Low & guzzle & with a rough surface & having a tight schedule \\
\hline
\end{tabular}

Note: Trained words in List 1 and List 2 are exposure controls in List 3 and List 4; and vice versa

\section{References}

Armstrong, B.C., \& Plaut, D.C. (2008). Settling dynamics in distributed networks explain task differences in semantic ambiguity effects: Computational and behavioral evidence. Paper presented at the Proceedings of the Cognitive Science Society.

Baayen, R.H., Davidson, D.J., \& Bates, D.M. (2008). Mixed-effects modeling with crossed random effects for subjects and items. Journal of Memory and Language, 59(4), 390-412. https://doi.org/ 10.1016/j.jml.2007.12.005

Bakker, I., Takashima, A., van Hell, J.G., Janzen, G., \& McQueen, J.M. (2014). Competition from unseen or unheard novel words: Lexical consolidation across modalities. Journal of Memory and Language, 73(0), 116-130. https://doi.org/10.1016/j.jml.2014.03.002
Balota, D.A., Yap, M.J., Cortese, M.J., Hutchison, K.A., Kessler, B., Loftis, B., ... Treiman, R. (2007). The english lexicon project. Behav Res Methods, 39(3), 445-459.

Bjork, R.A. (1994). Memory and metamemory considerations in the training of human beings.

Bjork, R.A., \& Kroll, J.F. (2015). Desirable difficulties in vocabulary learning. The American Journal of Psychology, 128(2), 241-252.

Bowers, J.S., Davis, C.J., \& Hanley, D.A. (2005). Interfering neighbours: The impact of novel word learning on the identification of visually similar words. Cognition, 97(3), B45-54. https://doi.org/10.1016/j.cognition. 2005.02.002

Brysbaert, M., \& New, B. (2009). Moving beyond kucera and francis: A critical evaluation of current word frequency norms and the introduction of a new and improved word frequency measure for 
american english. Behavior Research Methods, 41(4), 977-990. https://doi.org/10.3758/BRM.41.4.977

Brysbaert, M., Warriner, A.B., \& Kuperman, V. (2013). Concreteness ratings for 40 thousand generally known english word lemmas. Behavior Research Methods, 1-8.

Casenhiser, D.M. (2005). Children's resistance to homonymy: An experimental study of pseudohomonyms. Journal of Child Language, 32(2), 319-343.

Coutanche, M.N., \& Thompson-Schill, S.L. (2014). Fast mapping rapidly integrates information into existing memory networks. Journal of Experimental Psychology: General, 143(6), 2296-2303. https://doi. org/10.1037/xge0000020

Davis, M.H., \& Gaskell, M.G. (2009). A complementary systems account of word learning: Neural and behavioural evidence. Philosophical Transactions of the Royal Society of London. Series B, Biological Sciences, 364(1536), 3773-3800. https://doi.org/10.1098/rstb.2009.0111

Doherty, M.J. (2004). Children's difficulty in learning homonyms. Journal of Child Language, 31(1), 203-214.

Dudai, Y., Karni, A., \& Born, J. (2015). The consolidation and transformation of memory. Neuron, $88(1), 20-32$. https://doi.org/10.1016/j. neuron.2015.09.004

Fang, X., \& Perfetti, C.A. (2017). Perturbation of old knowledge precedes integration of new knowledge. Neuropsychologia, 99, 270-278. https://doi.org/10.1016/j.neuropsychologia.2017.03.015

Fang, X., Perfetti, C.A., \& Stafura, J. (2017). Learning new meanings for known words: Biphasic effects of prior knowledge. Language Cognition and Neuroscience, 32(5), 13.

Fernandez, R.S., Bavassi, L., Forcato, C., \& Pedreira, M.E. (2016). The dynamic nature of the reconsolidation process and its boundary conditions: Evidence based on human tests. Neurobiology of Learning and Memory, 130, 202-212. https://doi.org/10.1016/j.nlm.2016.03.001

Forcato, C., Fernandez, R.S., \& Pedreira, M.E. (2014). Strengthening a consolidated memory: The key role of the reconsolidation process. Journal of Physiology, Paris, 108(4-6), 323-333. https://doi.org/10. 1016/j.jphysparis.2014.09.001

Gaskell, M.G., \& Dumay, N. (2003). Lexical competition and the acquisition of novel words. Cognition, 89(2), 105-132. https://doi.org/10. 1016/s0010-0277(03)00070-2

Geukes, S., Gaskell, M.G., \& Zwitserlood, P. (2015). Stroop effects from newly learned color words: Effects of memory consolidation and episodic context. Frontiers in Psychology, 6, 278. https://doi.org/ 10.3389/fpsyg.2015.00278

Hino, Y., Lupker, S.J., \& Pexman, P.M. (2002). Ambiguity and synonymy effects in lexical decision, naming, and semantic categorization tasks: Interactions between orthography, phonology, and semantics. Journal of Experimental Psychology. Learning, Memory, and Cognition, 28(4), 686-713.

Kapnoula, E.C., \& McMurray, B. (2016). Training alters the resolution of lexical interference: Evidence for plasticity of competition and inhibition. Journal of Experimental Psychology. General, 145(1), 8-30. https://doi.org/10.1037/xge0000123

Kintsch, W., \& Mross, E.F. (1985). Context effects in word identification. Journal of Memory and Language, 24(3), 336-349.

Kuperman, V., Stadthagen-Gonzalez, H., \& Brysbaert, M. (2012). Age-ofacquisition ratings for 30,000 english words. Behavior Research Methods, 44(4), 978-990. https://doi.org/10.3758/s13428-012-0210-4

Landauer, T.K., \& Dumais, S.T. (1997). A solution to plato's problem: The latent semantic analysis theory of acquisition, induction, and representation of knowledge. Psychological Review, 104(2), 211240. https://doi.org/10.1037/0033-295x.104.2.211

Maciejewski, G., Rodd, J.M., Mon-Williams, M., \& Klepousniotou, E. (2018). The cost of learning new meanings for familiar words. https://doi.org/10.17605/OSF.IO/7YDKW
Mazzocco, M.M. (1997). Children's interpretations of homonyms: A developmental study. Journal of Child Language, 24(2), 441-467.

Mcclelland, J.L., Mcnaughton, B.L., \& Oreilly, R.C. (1995). Why there are complementary learning-systems in the hippocampus and neocortex - insights from the successes and failures of connectionist models of learning and memory. Psychological Review, 102(3), 419-457. https://doi.org/10.1037/0033-295x.102.3.419

Parks, R., Ray, J., \& Bland, S. (1998). Wordsmyth english dictionarythesaurus. University of Chicago.

Perfetti, C. A., \& Hart, L. (2002). The lexical quality hypothesis. In Precursors of functional literacy, 11, pp. 67-86.

Piercey, C.D., \& Joordens, S. (2000). Turning an advantage into a disadvantage: Ambiguity effects in lexical decision versus reading tasks. Memory \& Cognition, 28(4), 657-666. https://doi.org/10.3758/Bf03201255

Qiao, X., \& Forster, K.I. (2013). Novel word lexicalization and the prime lexicality effect. Journal of Experimental Psychology. Learning, Memory, and Cognition, 39(4), 1064-1074. https://doi.org/10. 1037/a0030528

Rodd, J.M., Berriman, R., Landau, M., Lee, T., Ho, C., Gaskell, M.G., \& Davis, M.H. (2012). Learning new meanings for old words: Effects of semantic relatedness. Memory \& Cognition, 40(7), 1095-1108. https://doi.org/10.3758/s13421-012-0209-1

Rodd, J.M., Cai, Z.G.G., Betts, H.N., Hanby, B., Hutchinson, C., \& Adler, A. (2016). The impact of recent and long-term experience on access to word meanings: Evidence from large-scale internetbased experiments. Journal of Memory and Language, 87, 16-37. https://doi.org/10.1016/j.jml.2015.10.006

Rodd, J.M., Lopez Cutrin, B., Kirsch, H., Millar, A., \& Davis, M.H. (2013). Long-term priming of the meanings of ambiguous words. Journal of Memory and Language, 68(2), 180-198. https://doi.org/ 10.1016/j.jml.2012.08.002

Roediger, H.L., 3rd, \& Butler, A.C. (2011). The critical role of retrieval practice in long-term retention. Trends in Cognitive Sciences, 15(1), 20-27. https://doi.org/10.1016/j.tics.2010.09.003

Storkel, H.L., \& Maekawa, J. (2005). A comparison of homonym and novel word learning: The role of phonotactic probability and word frequency. Journal of Child Language, 32(4), 827-853.

Storkel, H.L., Maekawa, J., \& Aschenbrenner, A.J. (2013). The effect of homonymy on learning correctly articulated versus misarticulated words. Journal of Speech, Language, and Hearing Research, 56(2), 694-707. https://doi.org/10.1044/1092-4388(2012/12-0122)

Tamminen, J., \& Gaskell, M.G. (2008). Newly learned spoken words show long-term lexical competition effects. The Quarterly Journal of Experimental Psychology, 61(3), 361-371. https://doi.org/10. 1080/17470210701634545

Tamminen, J., \& Gaskell, M.G. (2013). Novel word integration in the mental lexicon: Evidence from unmasked and masked semantic priming. The Quarterly Journal of Experimental Psychology, 66(5), 1001-1025. https://doi.org/10.1080/17470218.2012.724694

Tham, E.K., Lindsay, S., \& Gaskell, M.G. (2015). Markers of automaticity in sleep-associated consolidation of novel words. Neuropsychologia https://doi.org/10.1016/j.neuropsychologia.2015.03.025

van Kesteren, M.T.R., Krabbendam, L., \& Meeter, M. (2017). Integrating educational knowledge: Reactivation of prior knowledge during educational learning enhances memory integration

Van Petten, C., \& Kutas, M. (1987). Ambiguous words in context: An event-related potential analysis of the time course of meaning activation. Journal of Memory and Language, 26(2), 188-208.

Wang, H.C., Savage, G., Gaskell, M.G., Paulin, T., Robidoux, S., \& Castles, A. (2016). Bedding down new words: Sleep promotes the emergence of lexical competition in visual word recognition. Psychonomic Bulletin \& Review https://doi.org/10.3758/s13423-016-1182-7 